\title{
Lack of homology between two haloacetate dehalogenase genes encoded on a plasmid from Moraxella sp. strain B
}

\author{
Haruhiko Kawasaki, ${ }^{*}$ Ken Tsuda, Isao Matsushita and Kenzo Tonomura $\dagger$ \\ Department of Agricultural Chemistry, University of Osaka Prefecture, Sakai City, Osaka 591, Japan
}

(Received 4 November 1991; revised 17 February 1992; accepted 20 February 1992)

\begin{abstract}
Two genes encoding haloacetate dehalogenases, $\mathrm{H}-1$ and $\mathrm{H}-2$, are closely linked on a plasmid from Moraxella sp. strain B. H-1 predominantly acts on fluoroacetate, but H-2 does not. To elucidate the molecular relationship between the two enzymes, we compared their structural genes. Two restriction fragments of the plasmid DNA were subcloned on M13 phages and their nucleotide sequences were determined. The sequence of each fragment contained an open reading frame that was identified as the structural gene for each of the two dehalogenases on the basis of the following criteria; $N$-terminal amino acid sequence, amino acid composition, and molecular mass. The genes for $\mathrm{H}-1$ and $\mathrm{H}-2$, designated dehH1 and dehH2, respectively, had different sizes (885 bp and 675 bp) and $\mathrm{G}+\mathrm{C}$ contents $(\mathbf{5 8 . 3} \%$ and $\mathbf{5 3 \cdot 4} \%)$. Sequence analysis revealed no homology between the two genes. We concluded that the dehalogenases $\mathrm{H}-1$ and $\mathrm{H}-2$ have no enzyme-evolutionary relationship. The deduced amino acid sequence of the dehH1 gene showed significant similarity to those of three hydrolases of Pseudomonas putida and a haloalkane dehalogenase of Xanthobacter autotrophicus. The dehH2 coding region was sandwiched between two repeated sequences about $1.8 \mathrm{~kb}$ long, which might play a part in the frequent spontaneous deletion of dehH 2 from the plasmid.
\end{abstract}

\section{Introduction}

A number of soil micro-organisms have been isolated that can utilize short-chain haloalkanoic acids such as chloroacetate and 2-chloropropionate as their sole carbon source (Jensen, 1960; Kearney \& Kaufman, 1972). These micro-organisms have enzymes, known as 2-haloalkanoic acid dehalogenases, which catalyse the hydrolytic cleavage of halogen-carbon bonds by the following reaction (Goldman, 1965; Little \& Williams, 1971):

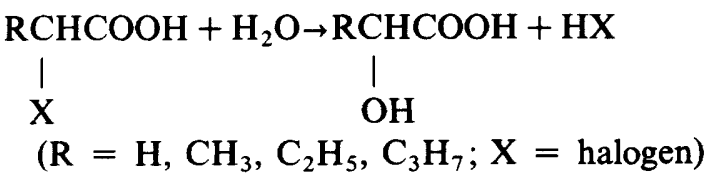

The dehalogenases are roughly classified into two groups on the basis of their substrate specificities. One group, termed haloacetate dehalogenase (EC 3.8.1.3),

\footnotetext{
* Corresponding author.

$\dagger$ Present address: Department of Food Technology, Fukuyama University, Fukuyama City, Hiroshima 729-02, Japan.

The nucleotide sequence data reported in this paper has been submitted to GenBank and has been assigned the accession numbers D90422 for dehH1 and D90423 for dehH2.
}

acts specifically on halogenated acetates to yield glycolate. The other group, termed 2-haloacid dehalogenase (EC 3.8.1.2), acts broadly on short-chain 2-haloalkanoic acids to produce 2-hydroxy acids. However, individual enzymes in each group exhibit different substratespecificities for carbon chain length, the halogen species, and the configuration of the substitute (Kawasaki, 1988). The diversity of the dehalogenases may result from selection for micro-organisms able to degrade a variety of novel halogenated compounds.

Enzyme evolution may be initiated by tandem duplication of a gene, followed by the accumulation of multiple mutations on either gene copy, which results in the creation of a modified enzyme (Rigby et al., 1974). If gene duplication is important in evolution, we would expect to find two similar enzymes in a cell at some stage of the evolutionary process. The nylon-oligomer-degrading enzymes found encoded on a plasmid by Okada et al. (1983) are an example of this. Although several bacteria have been isolated that contain two or more similar dehalogenases (Goldman et al., 1968; Weightman et al., 1979; Klages et al., 1983; Allison et al., 1983), the relationships between these enzymes have not been elucidated.

Moraxella sp. strain B, isolated from industrial wastewater, has two haloacetate dehalogenases, $\mathrm{H}-1$ and $\mathrm{H}-2$. 
These enzymes differ in halogen specificity; $\mathrm{H}-1$ acts better on monofluoroacetate than on monochloro- or monobromoacetate, but has little activity against monoiodoacetate. $\mathrm{H}-2$ acts on monochloro-, monobromo-, and monoiodoacetate, but not on monofluoroacetate. The two enzymes have been purified and characterized (Kawasaki et al., 1981a). The molecular masses estimated by SDS-PAGE were $33 \mathrm{kDa}$ for $\mathrm{H}-1$ and $26 \mathrm{kDa}$ for $\mathrm{H}-2$. Both enzymes were very sensitive to inhibition with thiol-blocking reagents such as $p$-chloromercuribenzoate. The other properties also indicated that $\mathrm{H}-1$ and $\mathrm{H}-2$ differed in molecular features, but not in catalytic features except for halogen specificity.

The genes for $\mathrm{H}-1$ and $\mathrm{H}-2$, designated dehHI and deh $H 2$, respectively, are borne on a conjugative plasmid, pUO1 (65 kb) (Kawasaki et al., 1981b), and have previously been cloned onto pBR322 in Escherichia coli (Kawasaki et al., 1984). The dehH1- and dehH2-coding restriction fragments that were cloned were located very close to each other on the restriction map of pUO1. Additionally, the two fragments formed a hybrid in a DNA-DNA hybridization test, implying that the fragments contained homologous sequences (Kawasaki, 1985). These findings suggested an enzyme-evolutionary relationship between $\mathrm{H}-1$ and $\mathrm{H}-2$.

Results presented in this paper show that $\mathrm{dehH} 1$ and $\operatorname{deh} \mathrm{H} 2$, and the encoded enzymes $\mathrm{H}-1$ and $\mathrm{H}-2$, show no sequence conservation, indicating that the two genes have not evolved from a common ancestral gene.

\section{Methods}

Bacteria, plasmids, phages, and growth conditions. Recombinant plasmids pBREF1 and pBRSG2 were used as sources for cloned dehH 1 and $\operatorname{deh} \mathrm{H} 2$, respectively. These plasmids were constructed previously by inserting a $3.9 \mathrm{~kb} E c o \mathrm{RI}$ restriction fragment of pUO1 (termed EcoRI-F fragment) and a $2 \cdot 1 \mathrm{~kb}$ Sall restriction fragment of pUO1 (termed SalI-G fragment) into pBR322 to generate pBREF1 and pBRSG2, respectively (Kawasaki et al., 1984). These plasmids were maintained and propagated in $E$. coli $\mathrm{C} 600$ (leu, thr, thi, hsdR, hsdM, $\operatorname{supE}$ ), which was grown in LB medium consisting of $10 \mathrm{~g}$ tryptone, $5 \mathrm{~g}$ yeast extract, and $5 \mathrm{~g} \mathrm{NaCl}$ per litre, $\mathrm{pH} 7 \cdot 3$.

Phages M13mp18 and mp19 and E. coli JM107 (Alac-pro, endA,

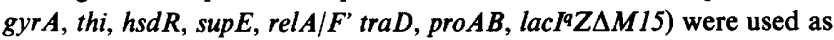
the vectors and host, respectively, for subcloning and sequencing. JM107 was maintained in M9 minimal medium (Maniatis et al., 1982) and grown for phage propagation in $\mathrm{YT}$ medium consisting of $8 \mathrm{~g}$ tryptone, $5 \mathrm{~g}$ yeast extract, and $5 \mathrm{~g} \mathrm{NaCl}$ per litre, $\mathrm{pH} 7 \cdot 3$.

DNA manipulations. Plasmid DNA and M13 replicative form (RF) DNA were isolated by the alkaline SDS method (Birnboim \& Doly, 1979). Isolation of M1 3 single-stranded DNA and subcloning into M13 vectors were performed as described by the M13 Cloning and Sequencing Handbook provided by Amersham. General procedures for DNA manipulations were according to Maniatis et al. (1982).

Construction of deletion subclones. The dehHl coding region is located within the overlap $(1.75 \mathrm{~kb})$ between the EcoRI-F fragment and the SalI-D fragment of pUO1, which is shown in the restriction map of

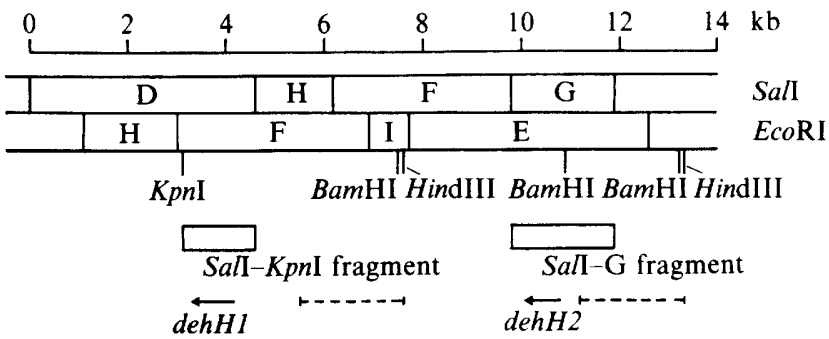

Fig. 1. Restriction map of the region encoding dehHl and $d e h H 2$ in the plasmid pUO1. Fragments yielded by EcoRI and $S a I I$ are shown by boxes with alphabetical letters. The $S a I-K p n I$ fragment and the $S a I I-$ $G$ fragment that were subcloned and sequenced are shown by boxes under the map. Arrows indicate locations and the direction of transcription of dehH1 and dehH2. Broken lines indicate locations of homologous sequences.

pUO1 in Fig. 1 (Kawasaki et al., 1984). However, to facilitate subsequent deletion with exonuclease III, a $1.5 \mathrm{~kb}$ segment from a $\mathrm{Sal}$ site to a KpnI site that contains a large portion of the overlap (Fig. 1) was subcloned for sequencing. The SalI-KpnI fragment was cut out from the pBREF1 DNA and inserted into two M13 vectors, $\mathrm{mp} 18$ and mp19. For the dehH 2 gene, the $2 \cdot 1 \mathrm{~kb}$ Sall-G fragment from pBRSG2 was subcloned in different orientations in $\mathrm{M} 13 \mathrm{mpl} 19$ vector.

A series of deletion subclones was constructed from each of the four subclones by use of the Kilo Sequence Deletion Kit (Takara Shuzo) according to the supplier's manual, essentially as described by Henikoff (1984). RF DNA was cleaved with two different restriction enzymes and digested with exonuclease III and mung bean nuclease, followed by ligation and transfection of JM107. Before transfection, the ligated DNA was treated with one of the restriction enzymes to linearize undeleted DNA.

Nucleotide sequencing. DNA was sequenced by the dideoxy chain termination method of Sanger et al. (1977) by use of the 7-DEAZA Sequencing Kit (Takara Shuzo) and $\left[\alpha^{-35} \mathrm{~S}\right] \mathrm{dCTP}\left(500 \mathrm{Ci} \mathrm{mmol}{ }^{-1}\right.$, 18.5 TBq mmol ${ }^{-1}$, New England Nuclear Corp.) as recommended by the supplier. Gel electrophoresis and autoradiography were done as described by the Amersham sequencing handbook. Sequence data were analysed and organized into a contiguous sequence with the aid of a sequence alignment program of the Genetic Data Processing Software, SDC-GENETYX (SDC).

Purification of dehalogenases from E. coli. Dehalogenases produced by $E$. coli clones harbouring $\mathrm{pBREF} 1$ and $\mathrm{pBRSG} 2$ were purified to homogeneity by the procedures described for the purification of $\mathrm{H}-1$ and H-2 from Moraxella sp. B (Kawasaki et al., 1981 a). Cells grown in LB broth containing $30 \mu \mathrm{g}$ ampicillin $\mathrm{ml}^{-1}$ were ruptured with an ultrasonic disintegrator. The enzymes were purified by ammonium sulphate fractionation, followed by three column chromatography steps using DEAE-Toyopearl 650M, Toyopearl HW-50 gel (Tosoh) and hydroxyapatite. The assays for the dehalogenases were as described previously (Kawasaki et al., 1981a).

Determination of the $N$-terminal amino acid sequence. The purified enzymes were desalted by dialysis, lyophilized, and dissolved in $1 \%$ (w/v) SDS solution at about $100 \mathrm{pmol}$ protein per $100 \mu \mathrm{l}$. Amino acid sequence analysis was carried out with an automatic peptide sequencer (Applied Biosystems Model 470A). Phenylthiohydantoin amino acid derivatives were identified by HPLC essentially as described by Hunkapiller \& Hood (1983). 


\section{Results and Discussion}

Characteristics of dehalogenases produced from $p B R E F 1$ and pBRSG2 in E. coli

Plasmids pBREF1 and pBRSG2 are known to express haloacetate dehalogenases in E. coli (Kawasaki et al., 1984). The dehalogenases purified from $E$. coli were shown to have the same mobility on SDS-PAGE and the same substrate specificity and catalytic properties as the corresponding enzymes from Moraxella (data not shown). These results confirmed that the dehalogenases produced from pBREF1 and pBRSG2 in $E$. coli were identical to $\mathrm{H}-1$ and $\mathrm{H}-2$, respectively.

The $\mathrm{N}$-terminal amino acid sequences determined with the enzymes from $E$. coli were Met-X-Phe-Pro-GlyPhe-Lys-Asn-Ser-Thr for H-1 and Met-Lys-Lys-Ile-GluAla-Ile-Ala-Phe-Asp for H-2. The second amino acid (X) of $\mathrm{H}-1$ was unclear.

\section{Nucleotide sequence of the dehHI gene}

A total of 26 deletion subclones derived from the recombinants of $\mathrm{M} 13 \mathrm{mpl} 18$ and 19 containing the $1.5 \mathrm{~kb}$
SalI-KpnI restriction fragment were subjected to DNA sequencing, and gave the fully overlapped sequence of both strands. A contiguous 1536 bp sequence determined for the SalI-KpnI fragment is shown in Fig. 2. Computer analysis of the sequence revealed an open reading frame (ORF) of $885 \mathrm{bp}$, beginning at the 413th nucleotide from the $5^{\prime}$ SalI end of the fragment. This ORF could be assigned to the $\mathrm{H}-1$ structural gene $(\operatorname{dehHI})$ based on the following arguments: The first ten amino acid residues deduced from the nucleotide sequence of the ORF were identical to the $\mathrm{N}$-terminal amino acid sequence determined for the $\mathrm{H}-\mathrm{l}$ enzyme. The amino acid composition and the molecular mass (33 $307 \mathrm{Da})$ for the deduced protein closely resembled those of the $\mathrm{H}-\mathrm{l}$ protein (Table 1).

A sequence, AGGAGA, located 6 bp upstream from the ATG initiation codon of the dehHI gene constituted a potential ribosome-binding sequence. Plasmid pBREF1 containing the EcoRI-F fragment expressed the deh $\mathrm{Hl}$ gene regardless of the orientation of the insert, while the recombinant containing the SalI-KpnI fragment did not express the dehHl without the aid of a promoter belonging to the vector. Such a case was a

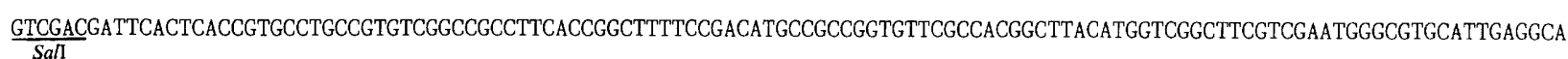

CTCCGGCCATACCTTGCCCCCTCGCAACGCACGGTGGGCACGCACGTCAATCTGAGCCACAGCGCCGCGACCCCGGTGGGCATGCAGGTTACGGCAGAGGTTGAGCTGATCGAGGTCGAA GGCAAGCGCTTGACATTCAAGGTCTTGTGCCGCGACGAGGTGGACGTGATCTGCGAGGGCCGGCACGAGCGCTTCATCGTTGAAGCGCAGCAGTTCATCAGACGAGTCACGTCCAAGGGT GAACGTGGCTGAGCTTTAGGGCTAAAGAAGTTGATCAACCCAGGAGACAAAA ATG GAC TTT CCA GGA TTC AAG AAC AGC ACC GTT ACC GTG GAT GGT GTG GAC $\begin{array}{lll}\text { SD } & \text { Met Asp Phe Pro Gly Phe Lys Asn Ser Thr Val Thr Val Asp G1y Va1 Asp }\end{array}$

ATC GCC TAC ACC GTA AGC GGC GAA GGC CCT CCG GTG CTG ATG CTG CAT GGG TTC CCG CAG AAC CGG GCC ATG TGG GCG CGC GTG GCT CCC
Ile Ala Tyr Thr Val Ser Gly Glu Gly Pro Pro Val Leu Met Leu His Gly Phe Pro Gln Asn Arg Ala Met Trp Ala Arg Val Ala Pro Ile Ala Tyr Thr Val Ser Gly Glu Gly Pro Pro Val Leu Met Leu His Gly Phe Pro Gln Asn Arg Ala Met Trp Ala Arg Val Ala Pro CAA CTC GCC GAG CAC CAT ACC GTG GTG TGT GCC GAC CTG CGA GGC TAT GGC GAT TCG GAC AAG CCC AAG TGC CTG CCG GAC CGG TCA AAC
G1n Leu Ala Glu His His Thr Val Val Cys Ala Asp Leu Arg Gly Tyr Gly Asp Ser Asp Lys Pro Lys Cys Leu Pro Asp Arg Ser Asn
50 TAC TCA TTC CGC ACG TTT GCC CAT GAC CAA CTC TGT GTG ATG CGC CAC CTG GGG TTC GAG CGC TTC CAC CTC GTC GGA CAT GAT CGC GGC
Tyr Ser Phe Arg Thr Phe Ala His Asp Gln Leu Cys Val Met Arg His Leu GIy Phe Glu Arg Phe His Leu Val Giy His Asp Arg Gly GGG CGT ACC GGT CAC CGC ATG GCG CTG GAT CAT CCC GAA GCG GTG CTG TCG CTG ACC GTC ATG GAC ATC GTG CCG ACG TAT GCG ATG TTC
Gly Arg Thr Gly His Arg Met Ala Leu Asp His Pro Glu Ala Val Leu Ser Leu Thr Val Met Asp Ile Val Pro Thr Tyr Ala Met Phe
110 ATG AAC ACC AAC CGT CTG GTT GCC GCT TCC TAC TGG CAT TGG TAT TTC CTG CAG CAG CCT GAG CCG TTC CCC GAG CAC ATG ATC GGT CAG Met Asn Thr Asn Arg Leu Val Ala Ala Ser Tyr Trp His Trp Tyr Phe Leu G1n Gln Pro Glu Pro Phe Pro Glu His Met Ile Gly Gln GAC CCG GAC TTC TTC TAT GAG ACC TGT TTG TTC GGG TGG GGG GCA ACC AAG GTG TCG GAC TTT GAC CAA CAA ATG CTG AAC GCA TAT CGC Asp Pro Asp Phe Phe Tyr Glu Thr Cys Leu Phe Gly Trp Gly Ala Thr Lys Val Ser Asp Phe Asp Gln Gln Met Leu Asn Ala Tyr Arg
170 GAG TCT TGG CGC AAC CCA GCC ATG ATT CAC GGC TCA TGC TCG GAC TAC CGC GCC GCC GCA ACA ATT GAC CTT GAA CAC GAT AGC GCG GAC Glu Ser Trp Arg Asn Pro Ala Met Ile His Gly Ser Cys Ser Asp Tyr Arg Ala Ala Ala Thr Ile Asp Leu Glu His Asp Ser Ala Asp ATC CAA CGG AAG GTG GAA TGC CCC ACC TTG GTT TTC TAC GGC TCA AAG GGG CAG ATG GGG CAG CTA TTC GAC ATA CCA GCC GAG TGG GCA Ile Gin Arg Lys Val Glu Cys Pro Thr Leu Val Phe Tyr Gly Ser Lys Gly Gln Met Gly Gln Leu Phe Asp Ile Pro Ala Glu Trp Ala
230 AAG CGC TGC AAC AAC ACT ACA AAC GCA TCT CTG CCA GGA GGC CAT TTC TTC GTG GAT CAG TTC CCC GCG GAA ACA TCA GAG ATT CTT TTG
Lys Arg Cys Asn Asn Thr Thr Asn Ala Ser Leu Pro Gly Gly His Phe Phe Val Asp Gln Phe Pro Ala Glu Thr Ser Glu Ile Leu Leu Lys Arg Cys Asn Asn Thr Thr Asn Ala Ser Leu Pro Gly Gly His Phe Phe Val Asp Gln Phe Pro Ala Glu Thr Ser Glu Ile Leu Leu
260 AAG TTT CTT GCT CGA AAC GGC TGA TACAGCAGGCGCTGCTTCGTTCTTGTGCCAGCTAAAAATAAACGCTAGAGAGAGGGCGAAGCAGTGTTGAAACCTGTTCTTGCGATTG Lys Phe Leu Ala Arg Asn Gly 290 CCTNTGGGTCGGTACTTGGCGGCCTGCTGCGATGGGGCTTGGGCCTCAAATTCAACAATCTGCTTCCCCATATTCCACCGGGAACATTAACTGTCGCATCCCGTACGATCATATGGTCGC TTGTGAAACAGGTGCGGGTGGGTCTGGTACC $\frac{1536}{\overline{\text { KpnI }}}$

Fig. 2. Nucleotide sequence of the SalI-KpnI fragment containing dehHI and its deduced amino acid sequence. The potential ribosomal binding site is underlined. The termination codon is indicated by an asterisk. Numbers under the amino acid sequence refer to the distances from the $\mathrm{N}$-terminal. Arrows represent a palindromic sequence located distal to the dehHl gene. 
Table 1. Comparison of the amino acid compositions and molecular masses deduced from the two ORFs with those of the $H-1$ and $H-2$ enzymes

\begin{tabular}{|c|c|c|c|c|}
\hline \multirow[b]{3}{*}{$\begin{array}{l}\text { Amino } \\
\text { acid }\end{array}$} & \multicolumn{4}{|c|}{ Number of residues per mol of enzyme } \\
\hline & \multicolumn{2}{|c|}{$\mathrm{H}-1$} & \multicolumn{2}{|c|}{$\mathrm{H}-2$} \\
\hline & $\begin{array}{c}\text { DNA } \\
\text { analysis* }\end{array}$ & $\begin{array}{c}\text { Protein } \\
\text { analysis } \dagger\end{array}$ & $\begin{array}{c}\text { DNA } \\
\text { analysis* }\end{array}$ & $\begin{array}{c}\text { Protein } \\
\text { analysis } \dagger\end{array}$ \\
\hline Gly & 22 & 24 & 16 & 17 \\
\hline Ala & 24 & 24 & 20 & 21 \\
\hline Val & 18 & 23 & 16 & 23 \\
\hline Leu & 22 & 22 & 20 & 21 \\
\hline Ile & 8 & 8 & 12 & 12 \\
\hline Ser & 15 & 15 & 13 & 12 \\
\hline Thr & 16 & 15 & 9 & 9 \\
\hline Asp & 21 & $>31$ & 12 & \rangle$_{10}$ \\
\hline Asn & 11 & $\int 31$ & 6 & \\
\hline Glu & 13 & 26 & 18 & $\gamma_{25}$ \\
\hline Gln & 12 & $\int^{20}$ & 8 & $\int^{20}$ \\
\hline Lys & 8 & 10 & 11 & 12 \\
\hline Arg & 17 & 17 & 12 & 11 \\
\hline Cys & 7 & 5 & 5 & 5 \\
\hline Met & 12 & 6 & 7 & 8 \\
\hline Tyr & 10 & 12 & 11 & 10 \\
\hline Phe & 20 & 19 & 10 & 10 \\
\hline His & 14 & 13 & 6 & 5 \\
\hline Trp & 6 & 8 & 4 & 5 \\
\hline Pro & 18 & 19 & 8 & 8 \\
\hline $\begin{array}{l}\text { Molecular } \\
\text { mass }(\mathrm{Da})\end{array}$ & 33307 & 33000 & 25345 & 26000 \\
\hline
\end{tabular}

* The total number of residues deduced from the ORF was 294 for $\mathrm{H}-1$ and 224 for $\mathrm{H}-2$.

† The number of residues was recalculated from previous data (Kawasaki, 1985) based on molecular masses of $33 \mathrm{kDa}$ for $\mathrm{H}-1$ and $26 \mathrm{kDa}$ for $\mathrm{H}-2$. amino acid composition and molecular mass of the dehH2 gene product corresponded closely to those of the H-2 enzyme (Table 1).

The dehH2 gene was preceded by the sequence GGAGA, the putative Shine-Dalgarno sequence, present $6 \mathrm{bp}$ upstream from the ATG initiation codon. A promoter that initiates transcription of dehH2 in E. coli must be present in the SalI-G fragment, because pBRSG2 and its derivative containing the Sall-G fragment, inserted in different orientations, expressed similar levels of H-2. The location of the promoter was analysed by progressively deleting the 5 -terminal region of the SalI-G fragment (Fig. 3). Deletion of $720 \mathrm{bp}$ from the $5^{\prime}$ terminus to a SmaI site did not obstruct the expression of dehH2, but further deletion of 275 bp up to a BamHI site resulted in failure to express the gene. When the remaining portion of the fragment was inserted downstream of one promoter carried by a vector, it could express the $d e h H 2$ gene. Therefore, the dehH 2 promoter working in $E$. coli should be located in the $275 \mathrm{bp}$ SmaI-to-BamHI region. A sequence TTGACA-(17 bases)-TAACCT present about $240 \mathrm{bp}$ upstream from the dehH 2 coding region was presumed to be the -35 and -10 sequences of the $d e h H 2$ promoter.

Plasmid pUOI expressed dehHI and dehH2 in several Pseudomonas hosts as well as in E. coli and Moraxella. However, no sequence similar to the known Pseudomonas promoters (Inouye et al., 1986) was found in either of the sequenced fragments. The deh $\mathrm{H} 1$ and $\mathrm{deh} \mathrm{H} 2$ genes were coded on the plasmid pUOI in the same transcription direction, that is, from right to left in the map given in Fig. 1.

\section{Comparison of dehH1 and dehH2}

The $\mathrm{G}+\mathrm{C}$ content of dehHl was $58.3 \%$ and that of deh 2 was $53.4 \%$. Both values were higher than that of the genomic DNA of genus Moraxella (40-47\%) (Bovre, 1984). As regards $G+C$ content alone, it seemed that the DNA of dehHl was rather similar to Pseudomonas DNA and the DNA of dehH2 was analogous to E. coli DNA. The two genes differed somewhat in their codon usage. Base preference for $\mathrm{G}$ and $\mathrm{C}$ in the wobble position was apparent in the dehHl gene $(72 \%)$, a phenomenon observed in a variety of organisms with high $\mathrm{G}+\mathrm{C}$ levels.

Comparison of the nucleotide sequences of $\mathrm{dehHl}$ and dehH2 with the aid of a computer program (SDCGENETYX) revealed no homology; the two genes showed no sequences 30 bases long with more than $50 \%$ identity. The amino acid sequences deduced from the genes also showed no significant similarity. Harr plot analysis (Staden, 1982) reconfirmed no homology between the two genes on the nucleotide sequence level and the amino sponded to that of the H-2 enzyme, and the predicted 

GGGCGCCCTGATGGAATTTAAAAAATCGAGCGCAGCACAATCCGCGACGAACATCGCCTTGCTTCGTCCTGGTGTGTCATCTTCAGCAAATGCGGAATCGTTGTCTGGGCTGATGAACAT AGTCTCGTCCTAACATTTGAGTAACCAGTATAAAAGCTATTGACAAGTTACGCCTCCGTGTCTAACCTACCCGGTGCTGCTGTAGGGTTACTTCATTGGGTACGACAGCATTCAGGATCT AGGCGAGCGGCCCGCGTGGGGGGGCGTTTTGCGCGGATCCGAGAGGCTAGATGTGACGGGTACAACGAAGATCGATTGCTGGCAGCGCGAGCGACGGCGTGCGGAAACGCTACAAGCAGC GAGCCATCGACCGACGCATATCTTAGAAAATGgagACATGA ATG AAG AAG ATC GAA GCC ATT GCA TTC GAC ATG TAC GGC ACC CTC TAC GAT GTG CAT SD 1 Lys Lys Ile Glu Ala Ile Ala Phe Asp Met Tyr Gly Thr Leu Tyr Asp Val His

TCG GTA GTG GAC GCA TGT GAG AAG CAG TAT CCA GGG AAG GGA AAA GAC ATC AGC GTC CTG TGG CGC CAA AAG CAA CTC GAA TAC GCT TGG 20 Val Val Asf Ala Cys Glu Lys Gln Tyr Pro Gly Lys Gly Lys Asp Ile Ser Val Leu Trp Arg Gin Lys Gln Leu Glu Tyr Ala Trp

TTG CGG TGC CTC ATG GGG CAG TAC ATC AAG TTC GAG GAG GCG ACA GCA AAT GCG TTG ACC TAC ACG TGC AAC CAG ATG AAG TTG GAT TGC

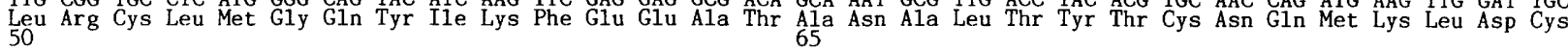

GAC GAG GGT TCG GCC ATG CGG CTC ACC GAG GAA TAT TTA CGC CTA AAA CCT TTT CCG GAG GTT CGA GGC GCA CTT CGA GCG CTG CGG CAG ${ }_{80}$ Asp Glu Gly Ser Ala Met Arg Leu Thr Glu Glu Tyr Leu Arg Leu Lys Pro Phe Pro Glu Val Arg Gly Ala Leu Arg Ala Leı Arg Gin

CGA GGA ATG CGG CTT GCG ATC CTG TCC AAC GGA TCG ACA GAA ACG ATT CAT GAC GTT GTT CAT AAC TCC GGC GTG GAG GGC GAG TTC GAG
Arg G1y Met Arg Leu Ala Ile Leu Ser Asn Gly Ser Thr Glu Thr Ile His Asp Val Val His Asn Ser Gly Val Glu Gly Glu Phe Glu Arg Gly Met Arg Leu Ala Ile Leu Ser Asn Gly Ser Thr Glu Thr Ile His Asp Val Val His Asn Ser Gly Val Glu Gly Glu Phe Glu CAT TTG ATC AGC GTG GAT TCC GCC CGG GCT TAC AAG CCC CAC CCT CTT GCC TAC GAA CTC GGA GAG GAA GCG TTC GGA ATA TCG CGC GAA
His Leu Ile Ser Val Asp Ser Ala Arg Ala Tyr Lys Pro His Pro Leu Ala Tyr Glu Leu Gly Glu Glu Ala Phe Gly Ile Ser Arg Glu
140

TCC ATT CTC TTT GTA TCG TCG AAT CCA TGG GAT GTA TCG GGA GCA AAA GCG TTC GGC TAT CAA GTC TGT TGG ATC AAT CGC TAT GGC TTT
Ser Ile Leu Phe Val Ser Ser Asn Pro Trp Asp Val Ser Gly Ala Lys Ala Phe Gly Tyr Gln Va1 Cys Trp Ile Asn Arg Tyr Gly Phe

GCG TTT GAC GAA CTG GGG CAG ACT CCT GAC TTC ACG GTT CCC GTG ATG GAT GCG ATT GTG CAT TTG ATC GCT GTA TGA GCCGATTACGGCAGGA Ala Phe Asp Glu Leu Gly Gln Thr Pro Asp Phe Thr Val Pro Val Met Asp Ala Ile Val His Leu Ile Ala Val
200

GGACAGAAGGAGAGGGGGCTGGTGCCCAAACACCGAGGCGCCTCGTCTCAAGGATCGTCAGACGCGCTTGTGTAAAGCCTGCTGCGGAAACAAAGAGGATAAATGCTTCCAGGCTCGTCT GACCAGCAGCGTAGCGTTCGCGCCGCCAGCCAGGNTGCTTCGACCTCGTCGCTGGCCTCGTAAGGCTGCGTCTAGTTGCGTTCCACGTAGCCCGGCGGAACGGCCAGCCCGCGTCGCGCC AGCAGGCGCAGCGCCTCGTCGTAGAGCGAAAGCGTGCGGTAGGCCGCGTCGAC 2105

Fig. 3. Nucleotide sequence of the Sall-G fragment containing dehH2 and its deduced amino acid sequence. The termination codon is marked by an asterisk. The putative Shine-Dalgarno sequence and the sequences similar to the -35 and -10 consensus of $E$. coli promoters are underlined. The region showing homology to that in the EcoRI-F fragment is overlined. Arrows represent palindromic sequences located distal to the dehH 2 gene.

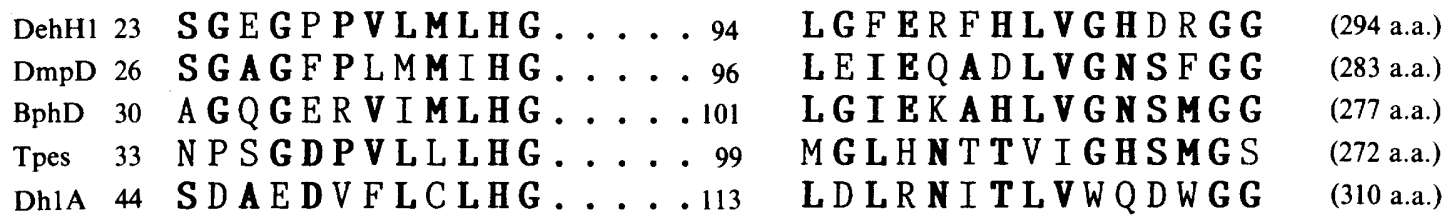

Fig. 4. Comparison of the predicted amino acid sequence of the $\mathrm{N}$-terminal region of $\mathrm{H}-1$ with those of three Pseudomonas hydrolases and a Xanthobacter dehalogenase. The sequence comparison was carried out using the program GENETYX. Well-conserved amino acids are emboldened. The number preceding each sequence refers to the amino acid number within each protein. Whole amino acid sequence length is shown in parentheses. DehH1, haloacetate dehalogenase H-1 (this work); DmpD, 2-hydroxymuconic semialdehyde hydrolase of $\boldsymbol{P}$. putida (Nordlund \& Shingler, 1990); BphD, 2-hydroxy-6-oxo-6-phenylhexa-2,4-dienate hydrolase of Pseudomonas sp. strain KKS102 (Kimbara et al., 1989); Tpes, tropinesterase of $P$. putida (Hessing, 1983); Dh1A, haloalkane dehalogenase of $X$. autotrophicus GJ10 (Janssen et al., 1989).

acid sequence level. The results suggest that the dehalogenases $\mathrm{H}-1$ and $\mathrm{H}-2$ have not descended from a common ancestral enzyme.

A computer-assisted search for nucleotide sequences homologous to dehH1 and $\operatorname{dehH2}$ in the nucleotide sequence data bases available to the GENETYX program gave no significant results. However, a search for amino acid sequences homologous to the deduced amino acid 
sequences of $d e h H 1$ and $d e h H 2$ in the protein data base sWISS-PROT yielded haloalkane dehalogenase (the gene product of $d h l A$, Janssen et al., 1989) of Xanthobacter autotrophicus and three hydrolases of Pseudomonas putida, i.e. 2-hydroxymuconic semialdehyde hydrolase (the gene product of $d m p D$, Nordlund \& Shingler, 1990), 2-hydroxy-6-oxo-6-phenylhexa-2,4-dienoate hydrolase (the gene product of $b p h D$, Kimbara et al., 1989) and tropinesterase (Hessing, 1983). The alignment of the amino acid sequences of these five proteins showed significant similarity in the $\mathrm{N}$-terminal region of each protein. Two highly conserved regions in all proteins are shown in Fig. 4. These similar $\mathrm{N}$-terminal regions (about 120-130 amino acids) might be common domains, which were derived from an ancestral Pseudomonas hydrolase.

The homology with other haloacetate dehalogenases of different bacterial strains isolated was previously examined by dot hybridization to the whole DNA extracted from the bacteria using the dehH1 DNA and dehH2 DNA as probes (Kawasaki et al., 1985). All 20 strains tested had DNA that hybridized with either of dehHl or dehH2 DNA; the 9 strains whose DNA hybridized with the dehHI DNA had $\mathrm{H}$-1-like dehalogenases acting preferentially on fluoroacetate, and the 11 strains containing DNA that hybridized with the dehH2 DNA had H-2-like dehalogenases that did not act on fluoroacetate. This suggests that bacterial haloacetate dehalogenases may be classified into two enzyme families, an $\mathrm{H}-1$ family and an $\mathrm{H}-2$ family.

\section{Repeated sequences preceding dehH1 and dehH2}

We wondered why the dehHI-containing EcoRI-F fragment had hybridized with the dehH2-containing SalI-G fragment (Kawasaki, 1985). This question was answered by sequencing and analysing the complete $3.9 \mathrm{~kb} E c o$ RI-F fragment (data not shown). Two fragments of EcoRI-F and SalI-G contained identical sequences of about $700 \mathrm{bp}$ upstream of the dehH1 and dehH2 coding regions (Fig. 3). Further sequencing of pUO1 DNA adjacent to these fragments revealed that the homologous sequence extended another $1100 \mathrm{bp}$ up to a HindIII site located in each flanking fragment as shown in Fig. 1.

The function of the homologous sequences and their relation to the dehalogenase genes are not clear. The two sequences were located on pUO1 repeatedly in the same orientation with the space of about $4 \mathrm{~kb}$ and held the dehH 2 coding region between. This region is deleted spontaneously from pUOl to form the deletion mutant pUO11 as reported previously (Kawasaki et al., 1983). The repeated sequences might possibly play a part in the deletion.
We thank Dr Hideki Yanagi of the Sumitomo Chemical Co. for analysis of the $\mathrm{N}$-terminal amino acid sequences of the dehalogenases. We also thank Hiroaki Sagawa and Kouichi Takatsu for purification of the enzymes, and Hidetoshi Kuriyama for analysis of the dehH 2 promoter. We are grateful to Professor C. M. Thomas (School of Biological Sciences, University of Birmingham, England) and the University of Edinburgh (Scotland) for helpful suggestions concerning the homologous amino acid sequences.

This work was supported in part by a grant-in-aid for Scientific Research from the Ministry of Education, Science, and Culture, Japan.

\section{References}

Allison, N., Skinner, A. J. \& COOPER, R. A. (1983). The dehalogenase of a 2,2-dichloropropionate-degrading bacterium. Journal of General Microbiology 129, 1283-1293.

BirnboIm, H. C. \& Doly, J. (1979). A rapid alkaline extraction procedure for screening recombinant plasmid DNA. Nucleic Acids Research 7, 1513-1523.

Bovre, K. (1984). Genus II. Moraxella. In Bergey's Manual of Systematic Bacteriology, vol. 1, pp. 296-303. Edited by N. R. Krieg and .J. G. Holt. The Williams \& Wilkins Co., Baltimore.

Goldman, P. (1965). The enzymatic cleavage of the carbon-fluorine bond in fluoroacetate. Journal of Bacteriology 240, 3434-3438.

Goldman, P., Milne, G. W. A. \& Keister, D. B. (1968). Carbonhalogen bond cleavage. Journal of Biological Chemistry 243, 428-434.

HeNIKOFF, S. (1984). Unidirectional digestion with exonuclease III creates targeted breakpoints for DNA sequencing. Gene 28, 351-359.

Hessing, J. G. M. (1983). Thesis, University of Leiden, Holland. Cited in the protein database SWISS-PROT (EMBL).

HunKaPILLER, M. W. \& HoOD, L. E. (1983). Analysis of phenylthiohydantoins by ultrasensitive gradient high-performance liquid chromatography. Methods in Enzymology 91, 486-493.

InOUYe, S., AsaI, Y., Nakazawa, A. \& NaKaZawa, T. (1986). Nucleotide sequence of a DNA segment promoting transcription in Pseudomonas putida. Journal of Bacteriology 166, 739-745.

Janssen, D. B., Pries, F., Ploeg, J., Kazemier, B., Terpstra, P. \& WITHOLT, B. (1989). Cloning of 1,2-dichloroethane degradation genes of Xanthobacter autotrophicus $\mathrm{GJ} 10$ and expression and sequencing of the dhlA gene. Journal of Bacteriology 171, 6791-6799.

JENSEN, H. L. (1960). Decomposition of chloroacetates and chloropropionates by bacteria. Acta Agriculturae Scandinavica 10, 83-103.

'KaWASAKI, H. (1985). Studies on plasmids determining bacterial haloacetate dehalogenases. Ph.D. thesis, University of Kyoto.

KAWASAKI, H. (1988). Dehalogenases and enzyme evolution (in Japanese). Bioscience and Industry 46, 16-23.

Kawasaki, H., Tone, N. \& Tonomura, K. (1981a). Purification and properties of haloacetate halidohydrolase specified by plasmid from Moraxella sp. strain B. Agricultural and Biological Chemistry 45, 3542.

Kawasaki, H., Yahara, H. \& Tonomura, K. (1981b). Isolation and characterization of plasmid pUO1 mediating dehalogenation of haloacetate and mercury resistance in Moraxella sp. B. Agricultural and Biological Chemistry 45, 1477-1481.

KaWASAKi, H., YahaRa, H. \& Tonomura, K. (1983). Cleavage maps of dehalogenation plasmid pUOl and its deletion derivative harbored in Moraxella sp. Agricultural and Biological Chemistry 47, 1639-1641.

Kawasaki, H., Yahara, H. \& Tonomura, K. (1984). Cloning and expression in Escherichia coli of the haloacetate dehalogenase genes from Moraxella plasmid pUO1. Agricultural and Biological Chemistry 48, 2627-2632.

Kawasaki, H., Masuda, M., Toyama, T. \& Tonomura, K. (1985). Uniformity of the genes of bacterial dehalogenases acting on fluoroacetate (in Japanese). Journal of the Chemical Society of Japan 1985, 1828-1831. 
Kearney, P. C. \& KaUfMan, D. D. (1972). In Degradation of Synthetic Organic Molecules in the Biosphere, pp. 166-189. Washington DC: National Academy of Science.

Kimbara, K., Hashimoto, T., Fukuda, M., Koana, T., Takagi, M., OISHI, M. \& YANO, K. (1989). Cloning and sequencing of two tandem genes involved in degradation of 2,3-dihydroxybiphenyl to benzoic acid in the polychlorinated biphenyl-degrading soil bacterium Pseudomonas sp. strain KKS102. Journal of Bacteriology 171, 2740 2747.

KlaGes, U., Krauss, S. \& Lingens, F. (1983). 2-Haloacid dehalogenase from 4-chlorobenzoate-degrading Pseudomonas spec. CBS3. Hoppe-Seyler's Zeitshrift für Physiologische Chemie 364, 529-535.

LitTle, M. \& Williams, P. A. (1971). A bacterial halidohydrolase. European Journal of Biochemistry 21, 99-109.

Maniatis, T., Fritsch, E. F. \& SambrooK, J. (1982). Molecular Cloning: A Laboratory Manual. Cold Spring Harbor, NY: Cold Spring Harbor Laboratory.

Nordiund, I. \& ShingleR, V. (1990). Nucleotide sequences of the meta-cleavage pathway enzymes 2 -hydroxymuconic semialdehyde dehydrogenase and 2-hydroxymuconic semialdehyde hydrolase from Pseudomonas CF600. Biochimica et Biophysica Acta 1049, 227-230.

Okada, H., Negoro, S., Kimura, H. \& Nakamura, S. (1983). Evolutionary adaptation of plasmid-encoding enzymes for degrading nylon oligomers. Nature, London 306, 203-206.

Rigby, P. W. J., Burleigh, B. D. \& Hartley, B. S. (1974). Gene duplication in experimental enzyme evolution. Nature, London 251, 200-204.

SANGER, F., Nicklen, S. \& Coulson, A. R. (1977). DNA sequencing with chain terminating inhibitors. Proceedings of the National Academy of Sciences of the United States of America 74, 5463-5467.

STADEN, R. (1982). An interactive graphics program for comparing and aligning nucleic acid and amino acid sequences. Nucleic Acids Research 10, 2951-2961.

Weightman, A. J., Slater, J. H. \& Bull, A. T. (1979). The partial purification of two dehalogenases from Pseudomonas putida PP3. FEMS Microbiology Letters 6, 231-234. 